11

الوعي البيئي لاي الزراع بمنطقتي قتدولة وقرناده بشعبية الجبل الأخضر- ليبيا

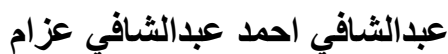

* قسم الإشاد الزراعي والتتمية الريفية. كلية الزرأعة-جامعة عمر المنتار - ليبيا

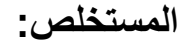

تهدف الدراسة بصفة أساسية إلي التعرف علي مستوي الوعي البيئي لدي الزراعي العي وتحديد

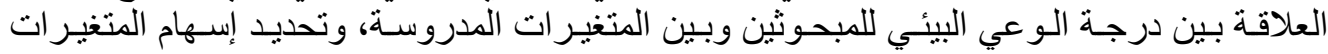

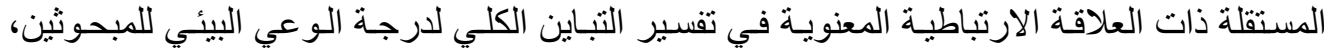

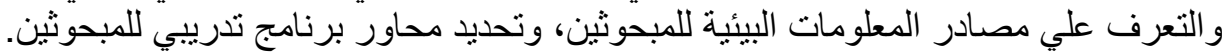

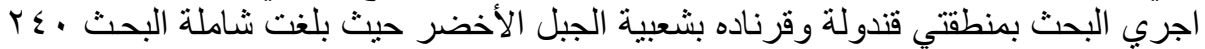

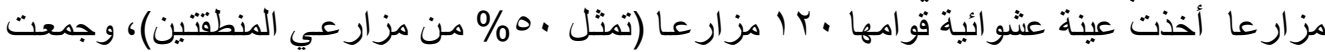

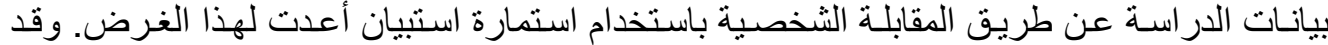

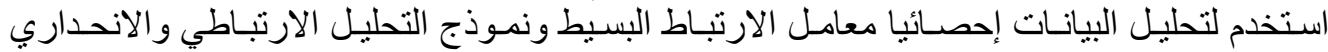

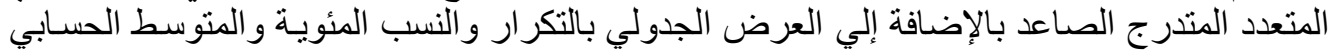

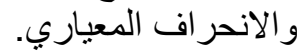

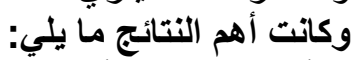

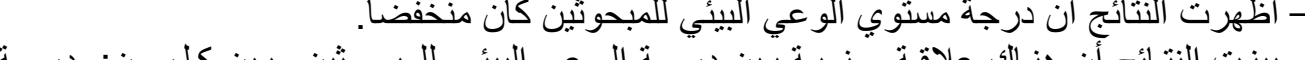

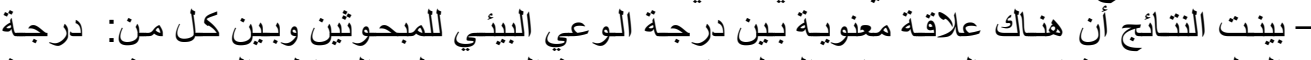

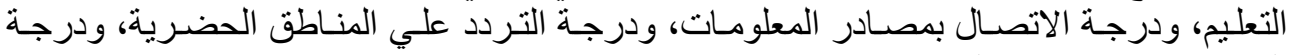
المشتاركة الاجتماعية و السن.

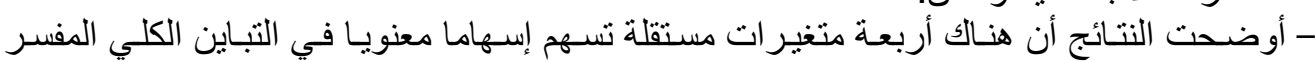

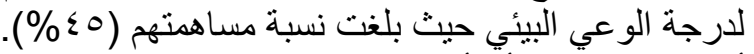

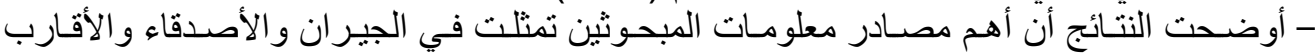

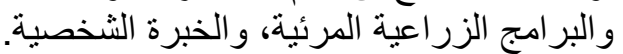

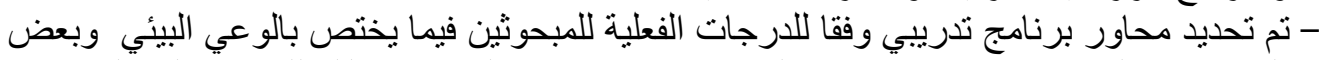

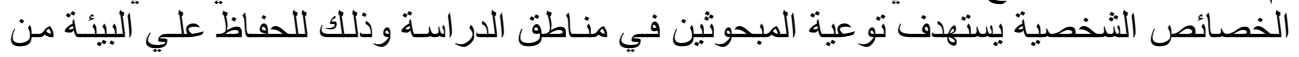
التلوث و الاستنز اف و التدهور.

المقدمة والمشكلة البحثية

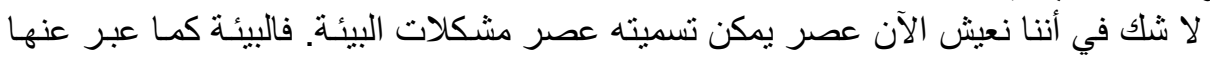

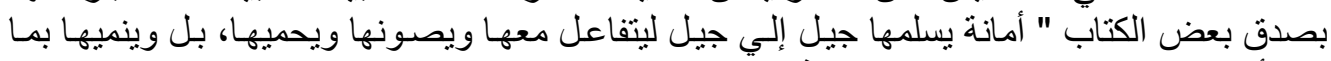

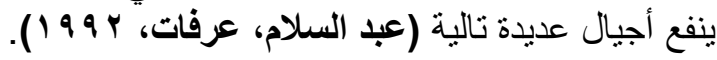

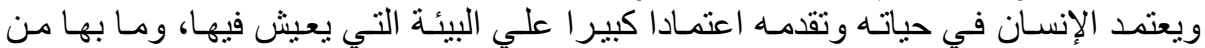

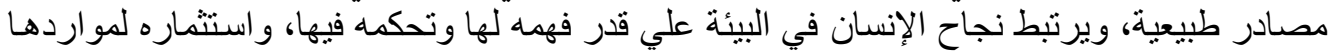

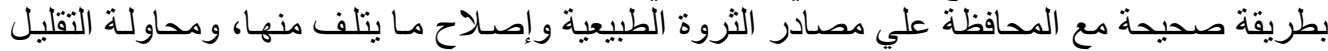

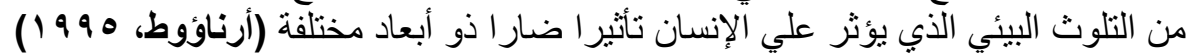

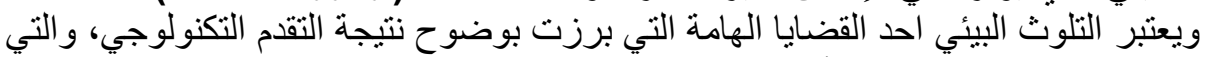

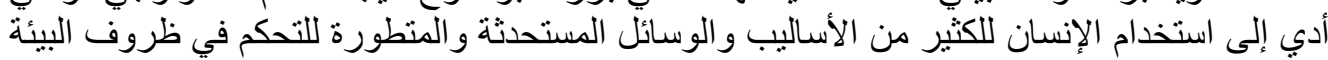

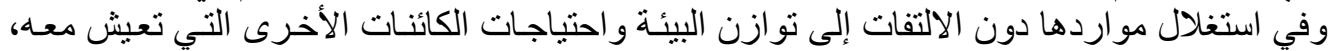

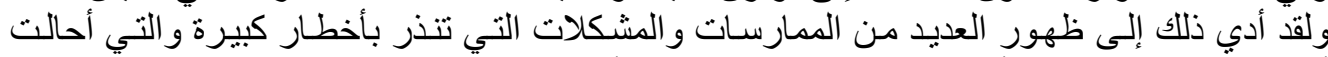

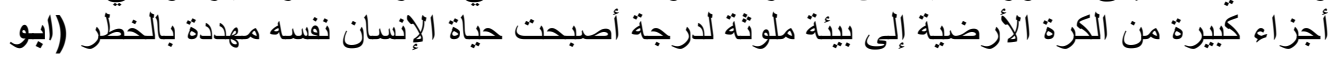

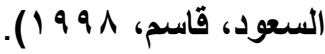

ونظر اللتأثير الخطير للمبيدات فان عديد من الجهات تدعو الزراع إلي خفض استخدامهم لها

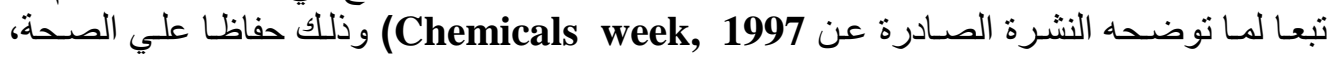

Fayoum J. Agric. Res. \& Dev., Vol.24, No.2, July, 2010 


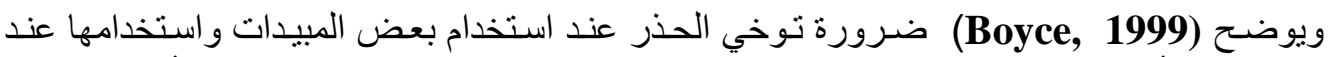

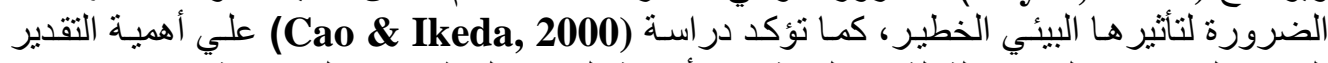

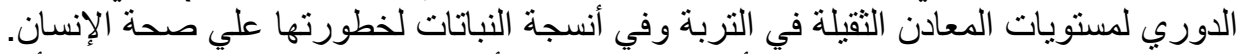

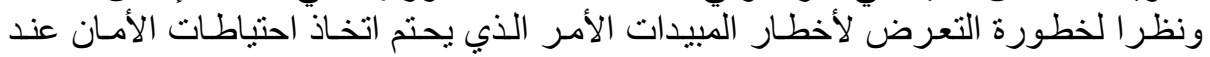

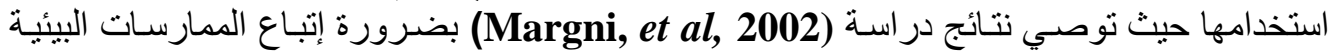

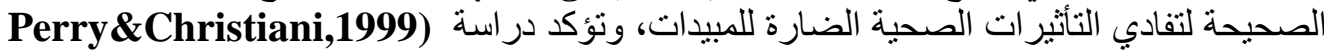
) علي أهمية إتباع السلوك الصحي الواقي من أخطار التعرض للمبرة المبيدات من خـالال الاهتمـام باستخدام الملابس و الأدوات الو اقية، كذللك تشير النشرة الصـادرة باسم (Ground Maintenance, 2002)

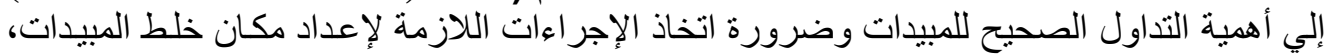

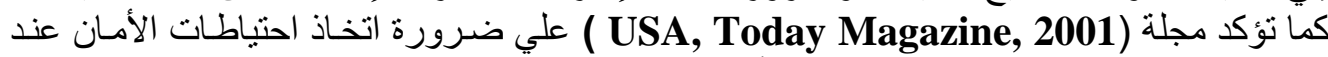

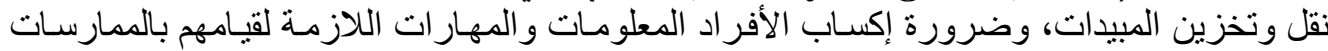
الصحيحة الخاصة بالوقاية من أخطار المبيدات.

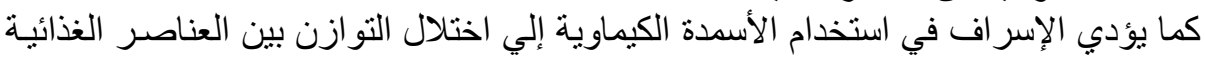

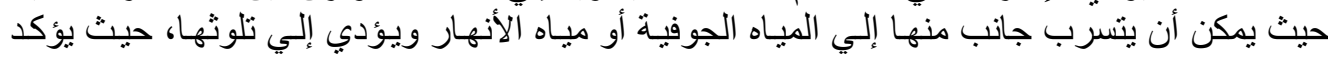
(Ockenden, 2002)

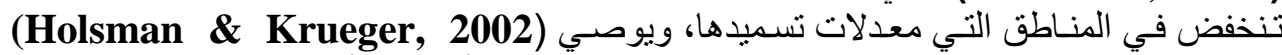

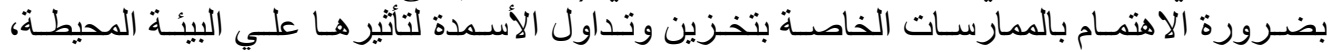

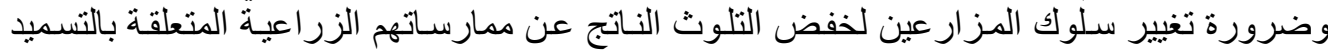

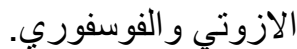

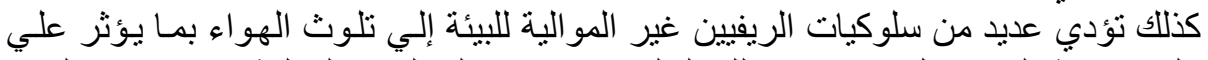

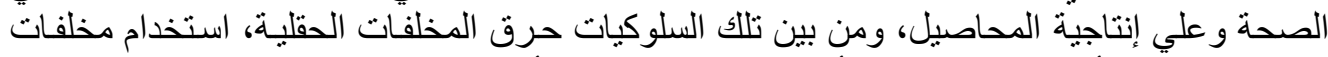

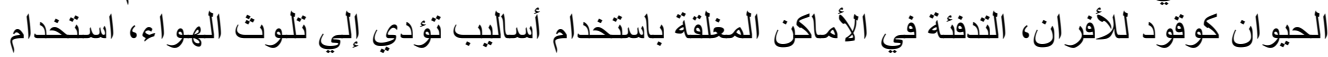

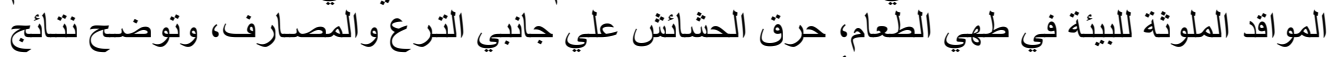

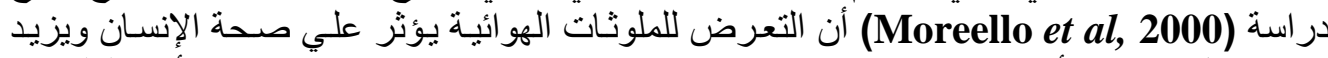

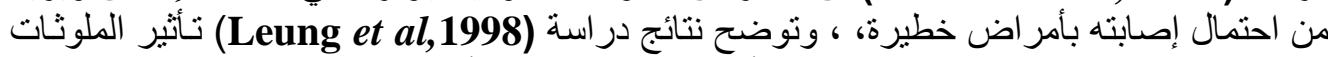

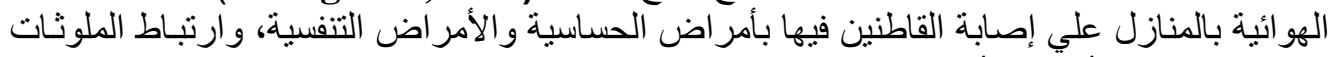

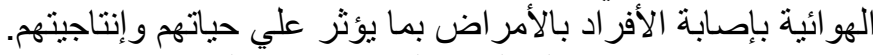

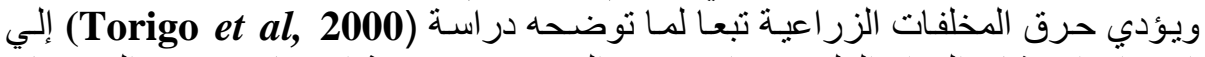

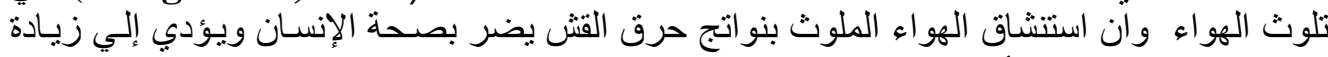

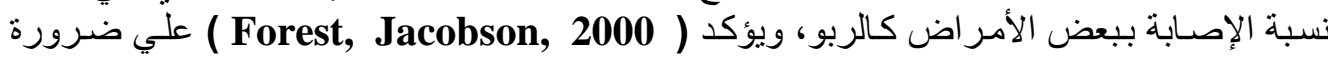

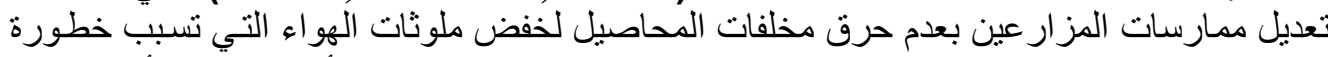

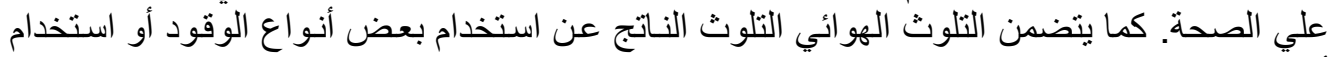

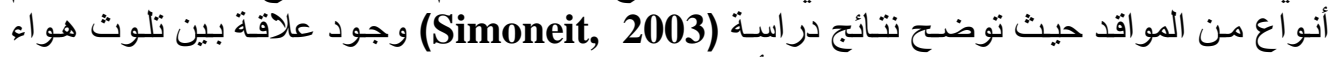
المنزل الناتج عن الوقود المستخدم بزيادة الأمر اض ناض التنفسية.

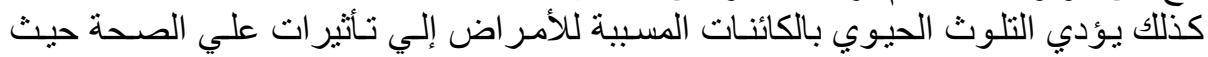

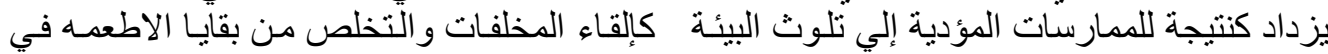

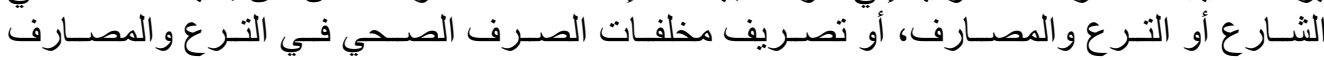

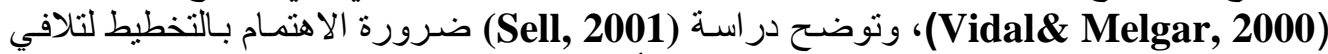

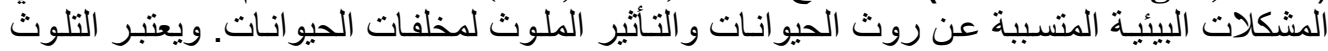

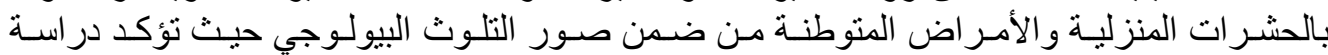

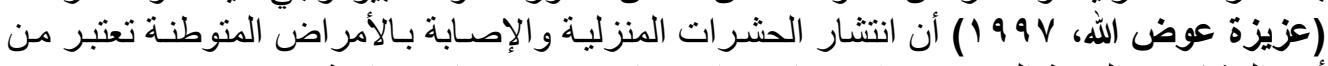

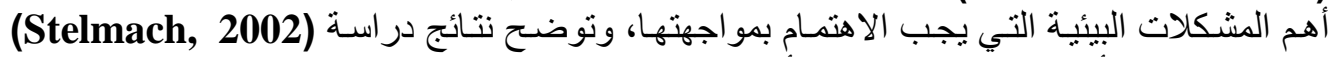

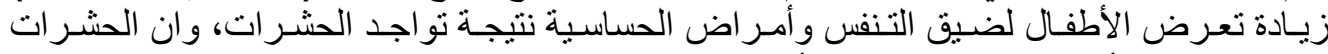

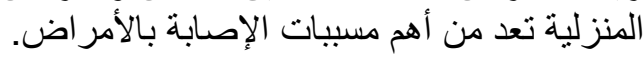

Fayoum J. Agric. Res. \& Dev., Vol.24, No.2, July, 2010 
ويعتبر التلوث الغذائي من أهم المشكلات المؤثرة على صحة الإنسان. ويمكن أن يحدث هذا التلوث خلال مرحلة الإنتاج أو النقل أو التسويق والعرض أو أو خلال مرحلة الإعداد و الطهي أو الحفظ

و هذا يستلزم تضافر الجهود للعمل على حلها. فقضية حماية البيئة وصيانتها هي قضية و التخزين.

الإنسان في المقام الأول وذلك لأنه المسئول عن تصاعد هذه المشكلات. وكذلك فهو المستفيد الأول

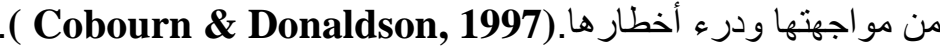

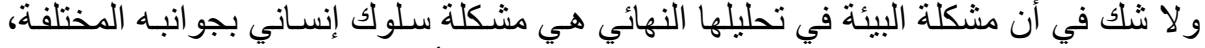

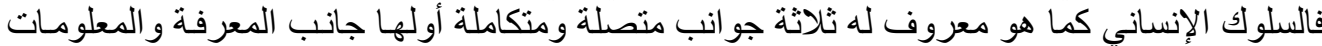

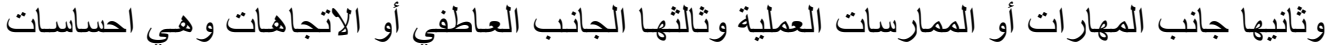
الفرد العاطفية سلبية كانت أم إيجابية نحو الأشياء أو الأشخاص.

وبالنظر إلي مشكلة البيئة كمشكلة سلوك إنساني ينضح أن لهذه المشكلة ثلاثة جو انب منطابقة

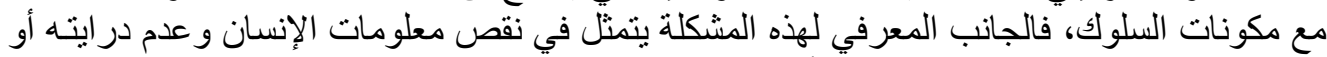

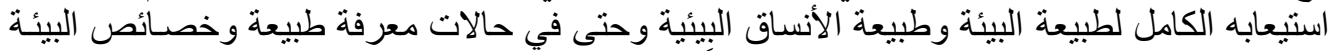

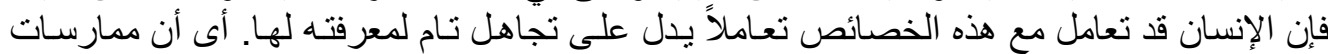

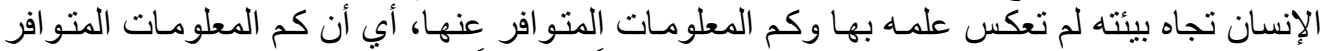

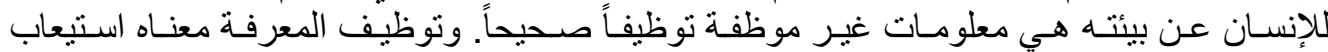

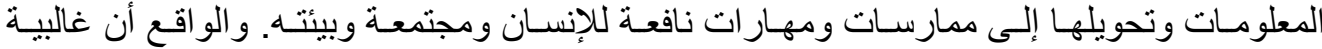

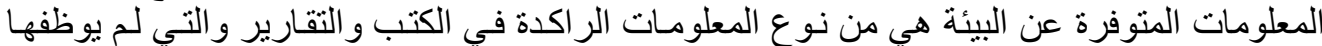

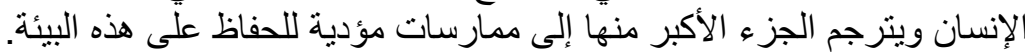

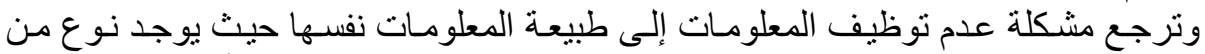

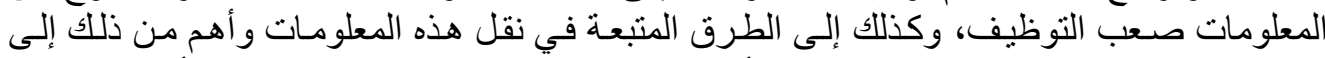

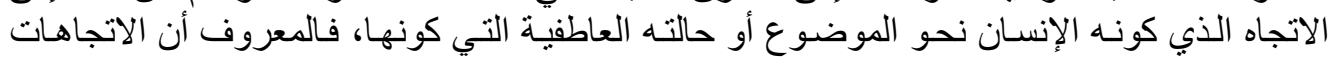

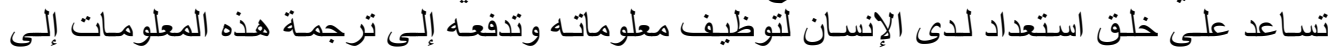

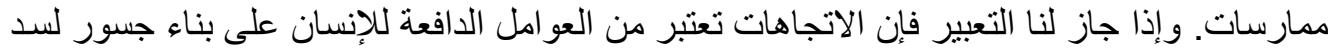

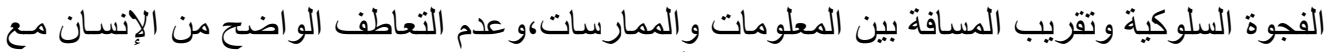

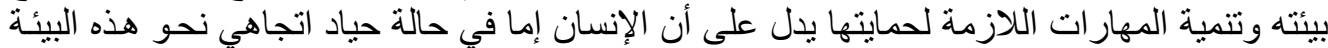

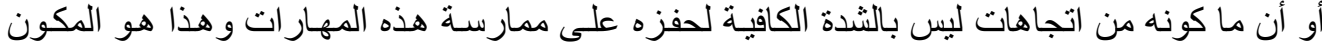
الثالث من مكونات السلوك البشري.

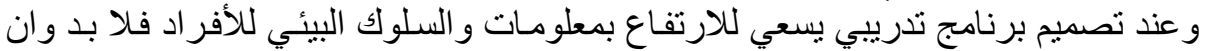

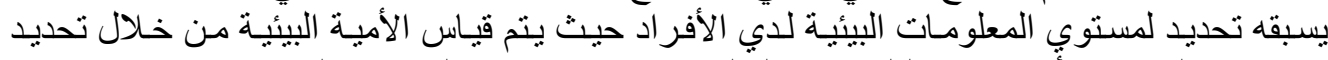

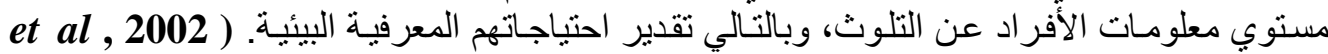

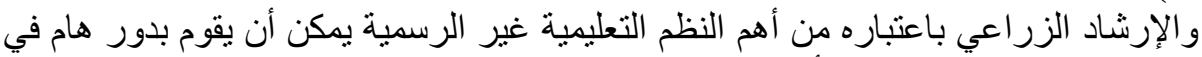

(Horrign,

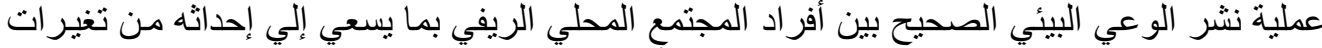

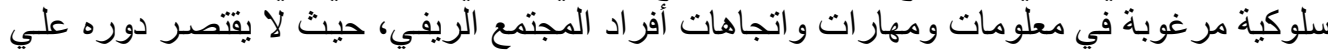

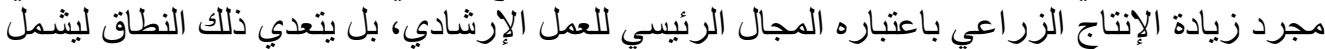

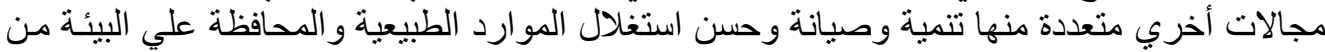

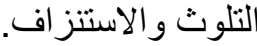

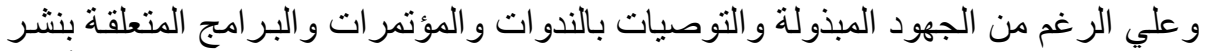

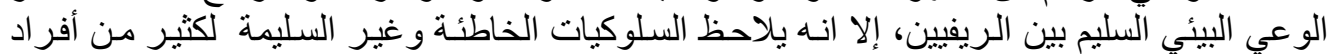

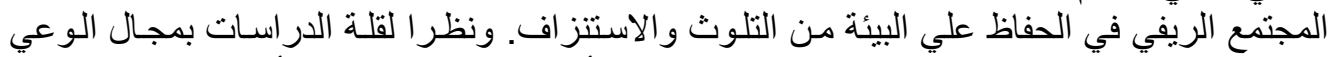

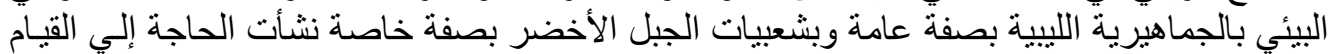

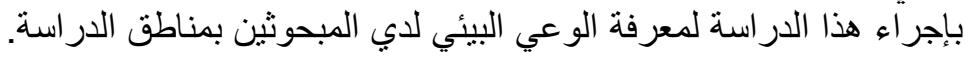

Fayoum J. Agric. Res. \& Dev., Vol.24, No.2, July, 2010 


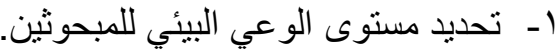

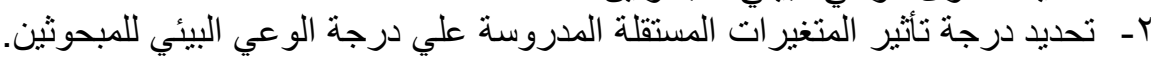
ك- ب- التعرف علي مصادر المعلومات البيئية للمبحوثين. ع - تحديد محاور برنامج تدريبي وفقا للارجات الفئئية الفحلية للمبحوثين للو عي البيئي.

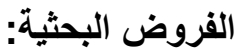

لتحقيق هدف البحث الثناني تم صباغة الفروض البحثنية التالية

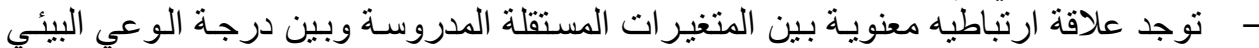
للمبحوثين وبين المتغير ات المئهين المستقلة المدروسة. - يوجد تأثير للمتغير ات المستقلة السابقة علي درجة الوعي البيئي للمبحوثين.

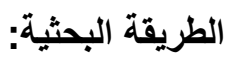

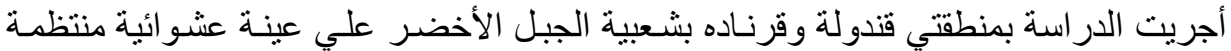

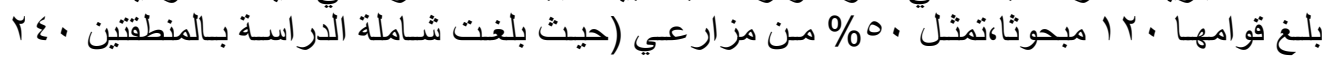

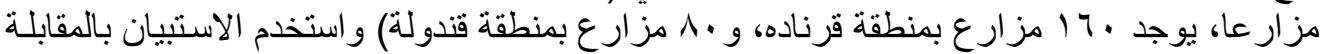

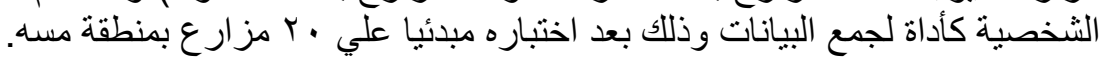

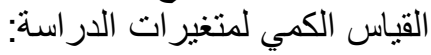

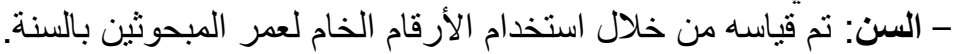

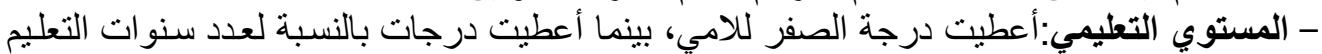

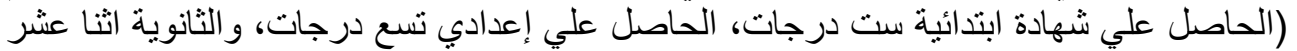

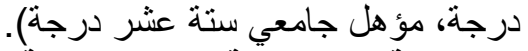

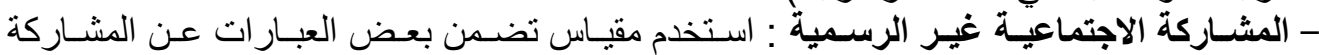

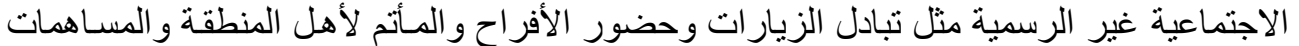

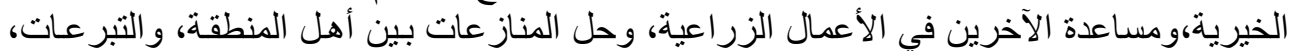

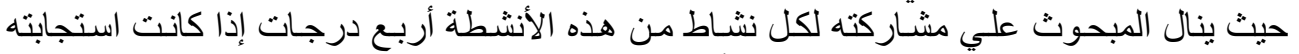

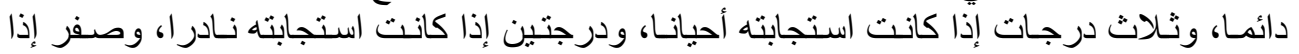

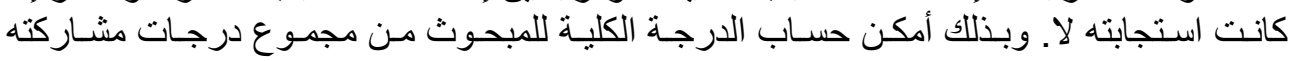
الاجتماعية غير الرسمية.

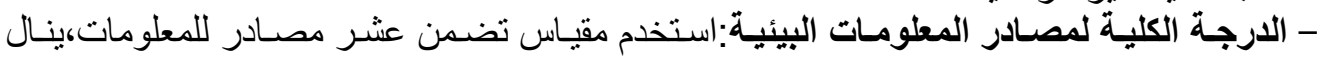

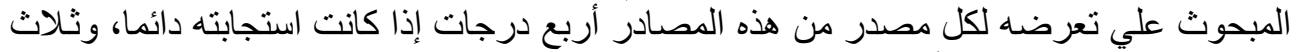

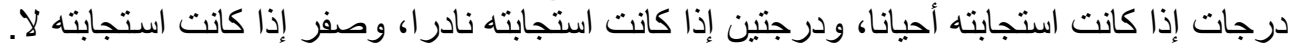

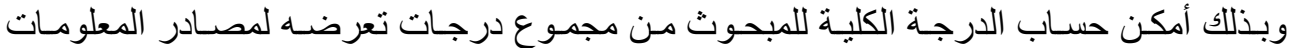
المحددة بالمقياس.

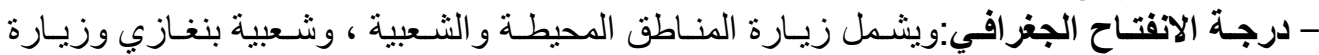

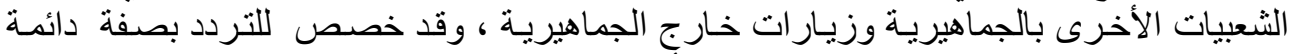

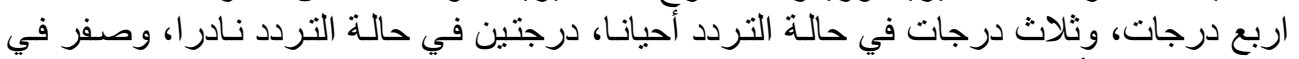

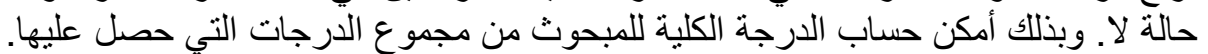

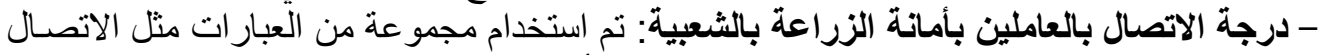

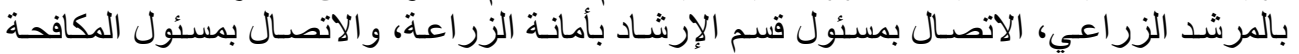

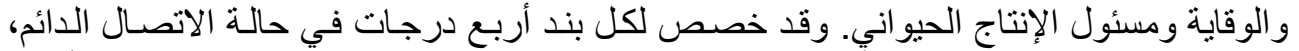

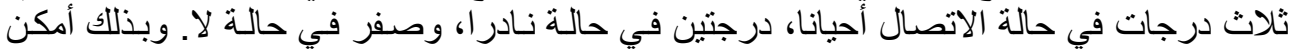

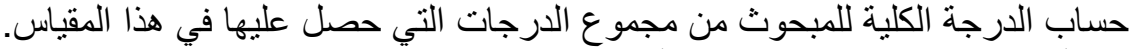

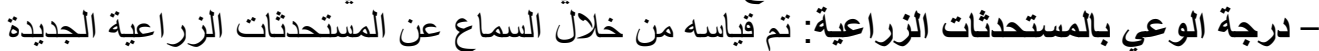

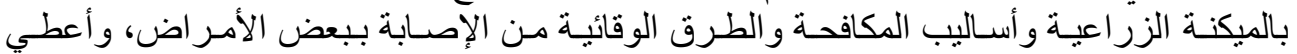

Fayoum J. Agric. Res. \& Dev., Vol.24, No.2, July, 2010 
10

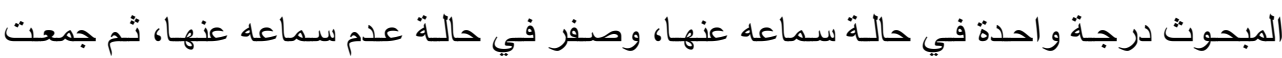

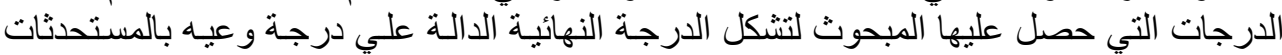
الزراعية.

- درجة الوعي البيئي للمبحوثين: استخدم مقياس مكون من · r عبارة تتعلق بدرجة و عي ومعرفة

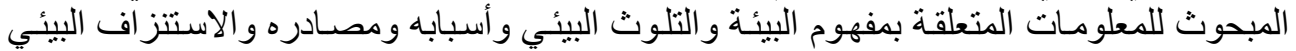

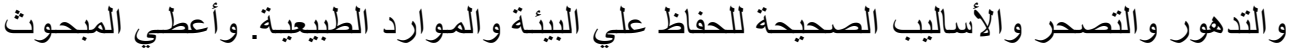

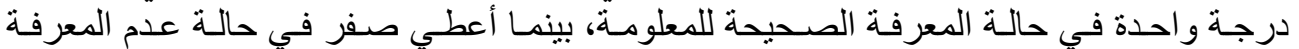

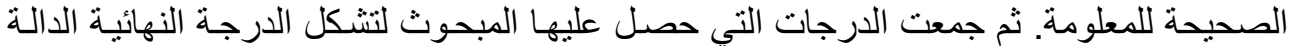

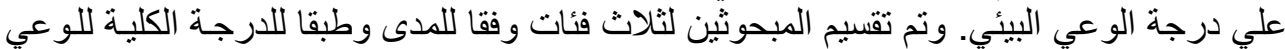

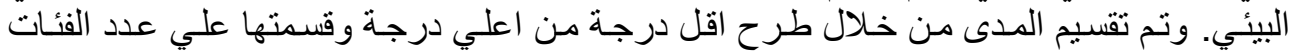
للحصول علي طول الفئة.

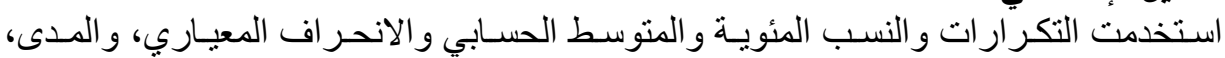
أدوات التحليل الإحصائي

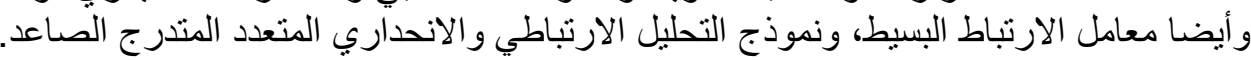

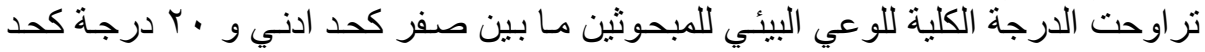

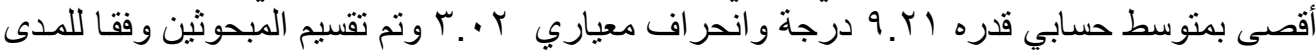

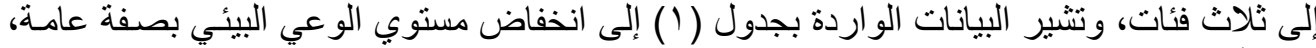

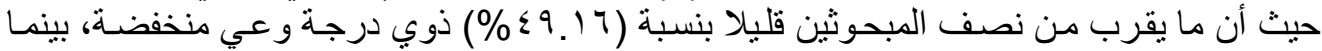

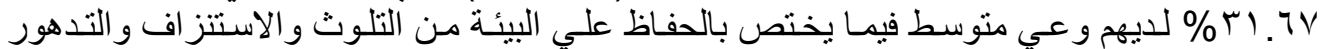

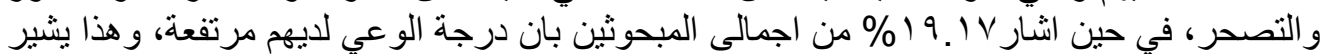

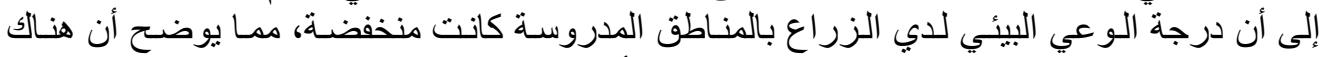

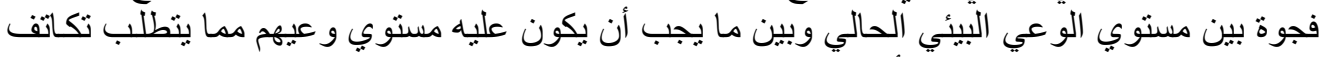

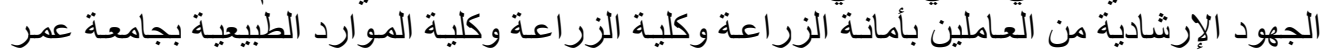

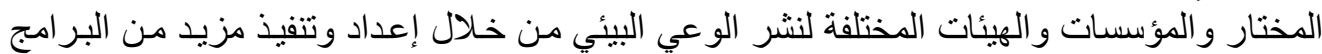

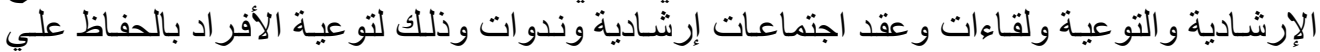

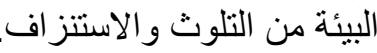

\begin{tabular}{|c|c|c|}
\hline$\%$ & 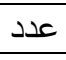 & درجة الوعي البيئي \\
\hline 49.16 & 59 & منخفضة (اقل من V درجات) \\
\hline 31.67 & 38 & متوسطة (V-ـ ا درجة) \\
\hline 19.17 & 23 & مرتفعة (0 ( درجة فأكثر) \\
\hline
\end{tabular}

جدول (r): قيم معامل الارتباط بين درجة الوعي البيئي وبين بعض المتغيرات المستقلة

\begin{tabular}{|c|c|}
\hline قيم معامل الارتباط & المتغير ات \\
\hline$* 0.202$ & السن \\
\hline$* * 0.521$ & درجة التعليح \\
\hline 0.023 & حجم الحيازة \\
\hline$* * 0.364$ & درجة المشاركة الاجتماعية \\
\hline 0.057 & درجة الاتصـال بالعاملين بأمانة الزر اعة \\
\hline$* * 0.431$ & درجة التردد علي المناطق الحضرية \\
\hline
\end{tabular}

Fayoum J. Agric. Res. \& Dev., Vol.24, No.2, July, 2010 
19

\begin{tabular}{|c|c|}
\hline$* * 0.398$ & درجة الاتصـال بمصادر المعلومات \\
\hline 0.098 & درجة الوعي بالمستحدثات الزر اعية \\
\hline 0.012 & عدد أفر اد الأسرة \\
\hline
\end{tabular}

ثانيا: العلاقة الارتباطية بين المتغيرات المستقلة المدروسة وبين درجة الوعي البيئي للمبحوثين:

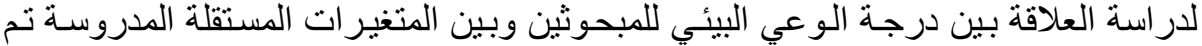

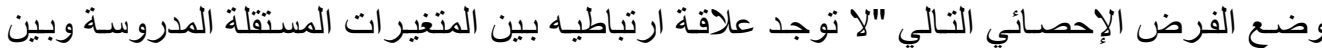

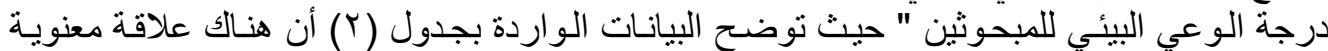

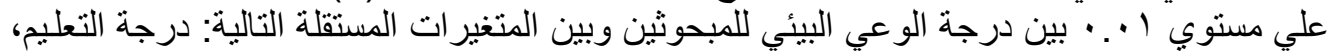

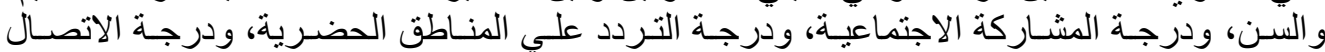

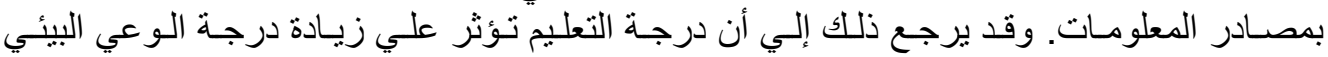

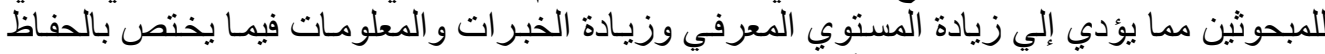

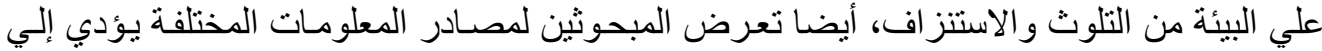

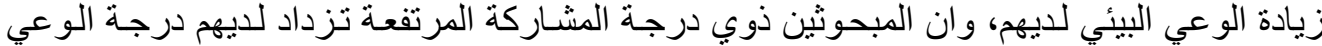

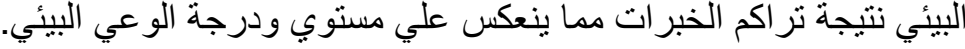

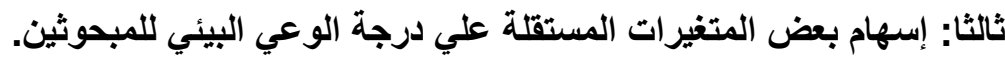

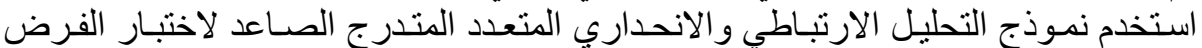

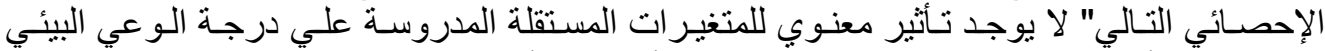

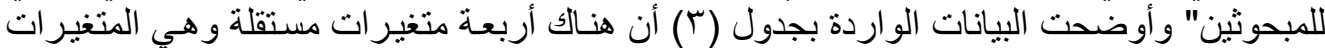

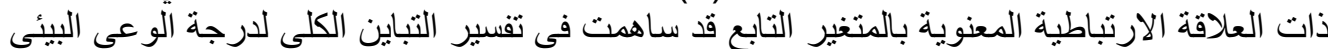

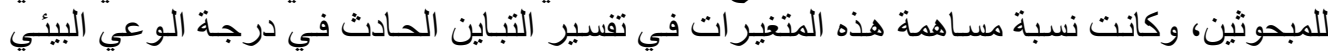

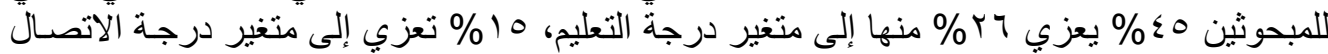

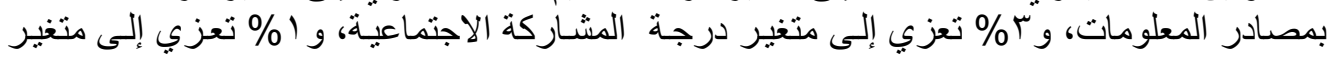

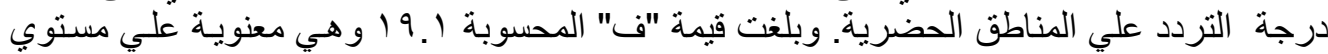

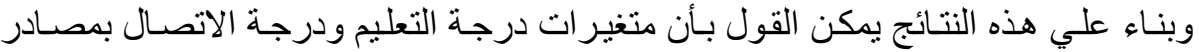

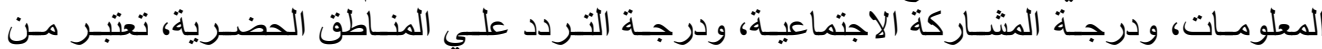

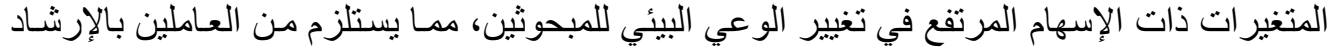

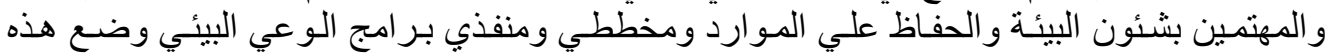

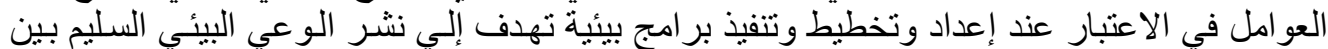
المزار عين و أفراد المجتمع الريفي.

جدول (ץ) التحليل الارتباطي والانحداري المتعدد المتدرج الصـاعد للعلاقة بين درجة الوعي البيئي

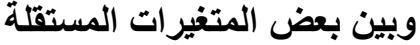

\begin{tabular}{|c|c|c|c|c|c|}
\hline معامل الانحدار & 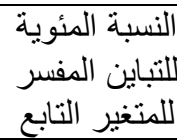 & المفر اكمية للتباين التنية التبية & | معامل الارنتباط & المتغير الداخل في & خطو ات التحليل \\
\hline$* * 4.651$ & 26 & 26.01 & $* * 0.521$ & درجة التعليم & الأوليوة \\
\hline$* * 2.980$ & 3 & 42.25 & $* * 0.364$ & درجة المشاركة & |الخطوة الثانية | \\
\hline
\end{tabular}

Fayoum J. Agric. Res. \& Dev., Vol.24, No.2, July, 2010 
iv

\begin{tabular}{|c|c|c|c|c|c|}
\hline$* * 3.258$ & 15 & 39.92 & $* * 0.398$ & بمصادر المعلومات الاتصال & الخطالثة الثطة \\
\hline$* * 1.470$ & 1 & 44.68 & $* * 0.431$ & المناطقة التردد علية & الخطوة الخعة \\
\hline
\end{tabular}

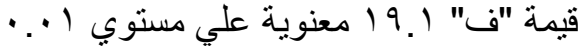

رابعا: مصادر المعلومات البيئية للمبحوثين:

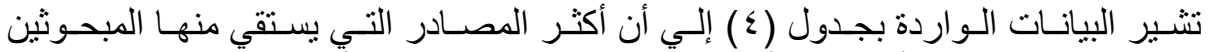

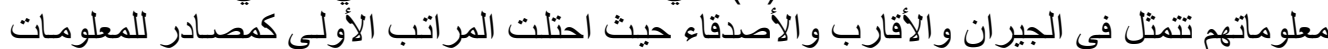

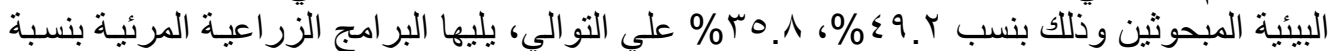

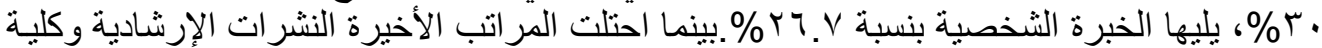

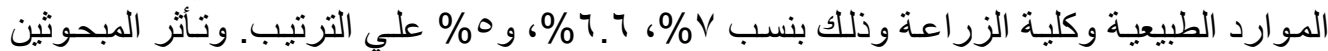

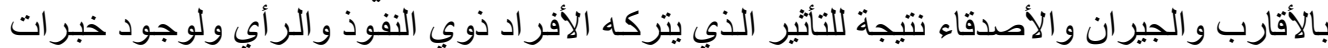

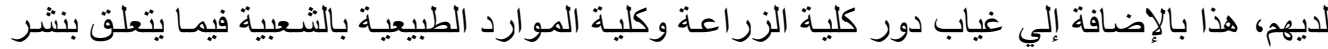

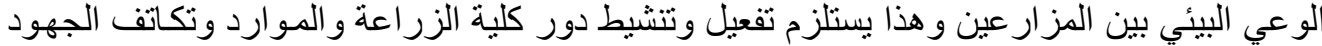

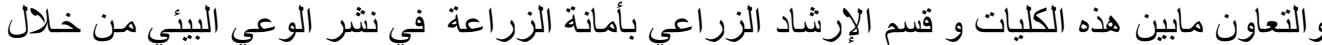

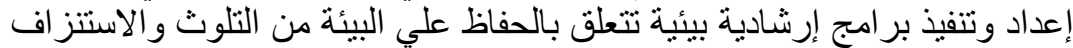

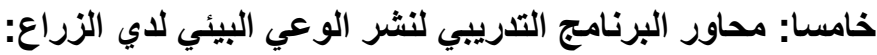

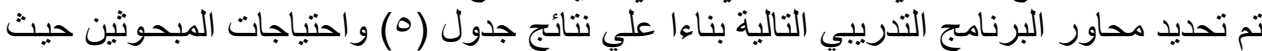

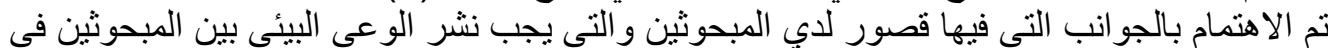

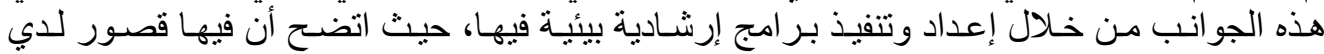

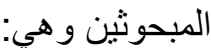
مفهوم البيئة وأَهمية الحفاظ علي الموارد البيئية من التلوث أو الاستنزاف أو التدهور.

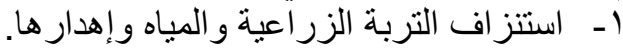
r- التلوث نتيجة استخدام الأسمدة و المبيدات الكيماوية.

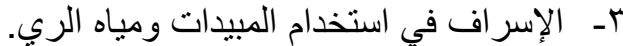

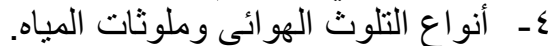
ــ التلوث نتيجة الطرق غير الصحيحة للتخلص من عبوات الأسمدة و المبيدات وبقايا المبيدات.

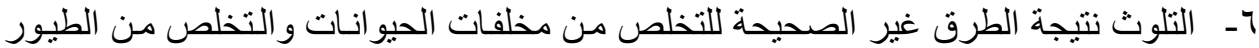
و الحيو انات الميته.

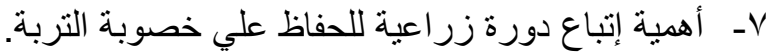

جلول (؛ ) توزيع المبحوثين وفقا لمصادر معلوماتهم البيئية

\begin{tabular}{|c|c|c|c|c|c|c|c|c|}
\hline \multicolumn{2}{|c|}{ ע } & \multicolumn{2}{|c|}{ نادرا } & \multicolumn{2}{|c|}{ أحيانا } & \multicolumn{2}{|c|}{ دائما } & المصادر \\
\hline $\begin{array}{c}\% \\
16.6\end{array}$ & عدد & $\%$ & & $\%$ & عدد & $\%$ & عدد & الصي (i) \\
\hline 14.2 & 17 & 27.5 & 33 & 22.5 & 27 & 35.8 & 43 & الأقارب و الأصدقاء \\
\hline 17.5 & 21 & 15.8 & 19 & 28.3 & 34 & 30 & 36 & البرامج المرئية "التليفزيون" \\
\hline 25.8 & 31 & 15 & 18 & 31.7 & 38 & 26.7 & 32 & الخبرة الثخصية \\
\hline 41.7 & 50 & 8.4 & 10 & 28.3 & 34 & 21.7 & 26 & أمانة الزر اعة \\
\hline 40 & 48 & 16.7 & 20 & 33.3 & 40 & 10 & 12 & المرشد الزراعي \\
\hline 40.8 & 49 & 30 & 36 & 20.8 & 25 & 8.4 & 10 & مركز البحوث الزراعية \\
\hline 40 & 48 & 25.8 & 31 & 26.7 & 32 & 7 & 9 & النشر ات الإرشادية \\
\hline
\end{tabular}

Fayoum J. Agric. Res. \& Dev., Vol.24, No.2, July, 2010 
11

\begin{tabular}{|c|c|c|c|c|c|c|c|c|}
\hline 58.3 & 70 & 19.2 & 23 & 15.8 & 19 & 6.6 & 8 & كلية الموارد الطبيعية بالثعبية الزراعة بالشعبية \\
\hline 49.2 & 59 & 25 & 30 & 20.8 & 25 & 5 & 6 & \\
\hline
\end{tabular}

\begin{tabular}{|c|c|c|c|c|}
\hline \multicolumn{2}{|c|}{ لا لا يعرف } & \multicolumn{2}{|c|}{ يعرف يع ي } & \multirow[t]{2}{*}{ المحسـاور } \\
\hline$\%$ & 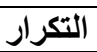 & $\%$ & 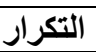 & \\
\hline 59.16 & 71 & 40.84 & 49 & ا ـ مفهوم البيئة \\
\hline 65 & 78 & 35 & 42 & r. بريقة استخدام المبيدات الكيماوية \\
\hline 55 & 66 & 45 & 54 & 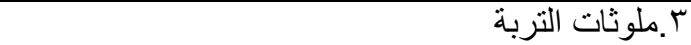 \\
\hline 67.5 & 81 & 32.5 & 39 & ـ ـأفضل طريقة للتخلص من عبو ات الأسمدة \\
\hline 58.34 & 70 & 41.66 & 50 & هـ.التخلص من عبوات المبيدات وبقايا المبيدات \\
\hline 51.67 & 62 & 48.33 & 58 & 7 أضر ار الإسر اف في استخدام الأسمدة الكيماوية \\
\hline 62.5 & 75 & 37.5 & 45 & Vا.أضر ار الإسراف في استخدام المبيدات \\
\hline 53.34 & 64 & 46.66 & 56 & ^ــأضر ار الإسراف في استخدام مياه الري \\
\hline 73.34 & 88 & 26.66 & 32 & q.أهية إتباع دورة زر اعية للحفاظ علي خصوبة التربة \\
\hline 60.84 & 73 & 39.16 & 47 & • ا.انسب طريقة للتخلص من المخلفات الزر اعية \\
\hline 35.84 & 43 & 64.16 & 77 & 1 (أفضل طريقة للتخلص من المخلفات المنزلية و القمامة \\
\hline 33.33 & 40 & 66.67 & 80 & r I أفضل طريقة للتخلص من روث المو اشي \\
\hline 36.66 & 44 & 63.34 & 76 & r أ.أسباب التصحر ببعض مناطق شعبية الجبل الأخضر \\
\hline 53.33 & 64 & 46.67 & 56 & ـ ا ـانسب طريقة للتخلص من الطيور و الحيو انات الميتة \\
\hline 25 & 30 & 75 & 90 & 10.فو ائد الغطاء النباتي بالثعبية \\
\hline 41.67 & 50 & 58.33 & 70 & ا 7 أضر ار الرعي الجائر \\
\hline 16.67 & 20 & 83.33 & 100 & V ا. انسب مكان لإعداد الطعام \\
\hline 20.84 & 25 & 79.16 & 95 & ^1 ـ فو ائد تثجير الثو ارع بشعبية الجبل الأخضر \\
\hline 65 & 78 & 35 & 42 & 19 ـملوثات المباه \\
\hline 58.34 & 70 & 41.66 & 50 & •r.أنواع التلوث الهوائي \\
\hline
\end{tabular}

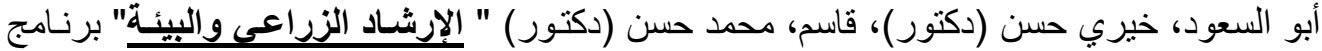

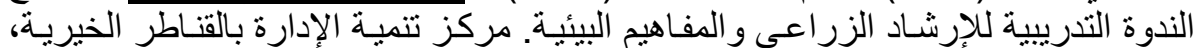

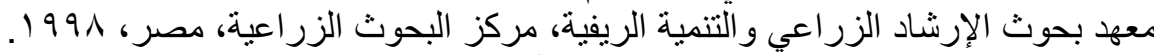

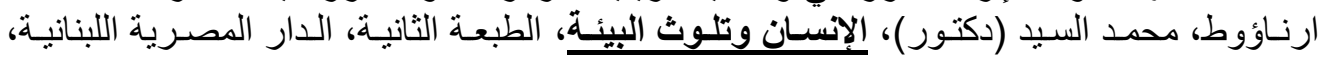

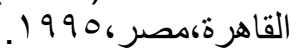

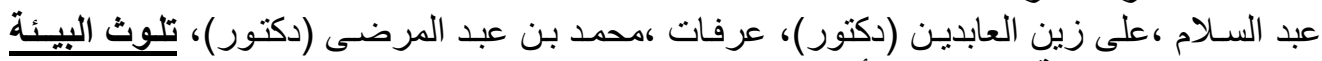

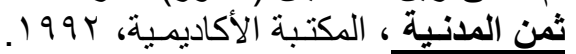

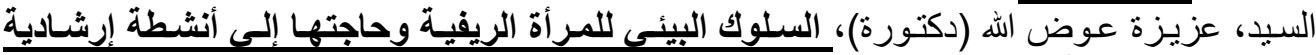

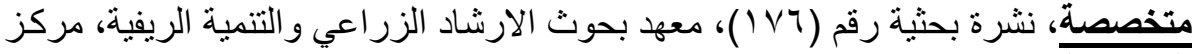

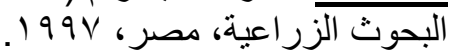

Bouman, M.A., Castaeda, A.R., Bhuiyan. S.I., "Nitrate and pesticide contamination under rice - basesd cropping systems: past and current evidence from the Philippines", agriculture ecosystems \& environment, Vol. 92, N., 2/3, November, 2002, academic search premier database.

Boyce, Nell, "breeding trouble" new scientists, Vol. 163. N. 2193, 7/3/1999.. academic search premier database.

Fayoum J. Agric. Res. \& Dev., Vol.24, No.2, July, 2010 
19

Cao, Hongbin, Ikeda, Saburo, "Exposure Assessment of heavy metals resulting from farmland application af wastewater sludge in Tianjin, china: risk analysis an official publication of the society for risk analysis, vol 20, Issu 5, Oct, 2000. Business source premier database.

Chemical Week, "Minor uses protected" Vol. 159, Issue 35, 9/1997, academic search premier database.

Coboun, John, Donaldson, Sue, "Reaching A New Audience", Journal Of Extension, Vol.35, N.1, February 1997.

Grounds Maintenance, "Handle with care", Vol. 37, N.3. March, 2002, academic search premier database.

Forrest, L.H., Jacobson, W.A., "Rice straw as a lignocellulosic resource: collection, processing, transportation and environmental aspects" biomass and bioenergy, Vol., 18, N. 5. 2000.

Holsman, Robert, h, Krueger, David, The long and short of groundwater education for Michigan farmers, Journal of extension, Vol., 40, N., 1, February, 2002.

Horrign, Leo, Lawrence Robert S., Walker, Polly, "How Sustainable Agriculture Can Address Environment And Human Health Harms Of Industrial Agriculture", Environment Health Perspectives, Vol. 110, Issue 5 , May 2002. Academic Premier Database

Leung, Lam, Chan, Lee, Chan, Pang, Lay, "Indoor environment of residential homes in Hong Kong- relevance to asthma, clinical experimental allergy, Vol., 28, issue 5, may 1998, academic search premier database.

Margni, M, Rossier, D., Crettaz, P., "Life cycle impact assessment of pesticides on human healthb and ecosystems, Agriculture, Ecosystems \& Environment, Vol., 93, N.1-3, Dec 2002. academic search premier database.

Morello, F, Rachal, A., Woodruff, T., Tracey, J., Axelrad, Daniel A.," Air toxics and health risks in California: the public health implecations of outdoor, risk analysis, Vol., 20, N. 2, Apr., 2000, academic search premier database.

Ockenden, Karma, "Industry will be left paying clean - up bill" utility week, Vol., 18, N. 23, 12/6/2002. Academic Search premier database.

Perry, Melssa, J., Christiana, David C., "herbicide and insecticide exposure among dairy farm pesticide" American journal of public health, Vol. 89, N.,7, July 1999, academic search premier database.

Repley, Richard, "Ottawa ethanol plant raises hope clearer skies", J. of business, Vol.,15, N.2, 1/11/2001. Regional business news database.

Rudolph, D.L., Goss, M.J., Barry, D.A.J., "Contamination in otario farmstead its association with agriculture", J. of Contaminatant hydrology, Vol., 32, N.3/4., 8/1/ 1998. academic search premier database.

Fayoum J. Agric. Res. \& Dev., Vol.24, No.2, July, 2010 
r.

Simoneit, bernd R,T, Rushdi,A,L.,Bin Abas, M, R.,'Environmental science \&technology", Vol., 37, N.1, 1/1/2003. academic search premier database.

Smith, Kirk, R., Mccracken, John, "Emissions and efficiency of improved woodburning cookstoves in highland guatimala" Environmental international, Vol.24, N,7, Oct. 1998. Academic Search premier database.

Torigoe, Katsumi, "Influence of emissions from rice straw burning on bronchial asthma in children" pedriatics international, Vol., 42, N.2, Apr. 2000. Academic Search premier database.

USA Today magazine, "Assuring security for dangerous pesticides", Vol. 130, N.2679, December 2001. Academic Search premier database.

Varlamoff,Susan, "homeowners and their choice of information sources about gardening" J. of Extension, Vol., 40, N.3, June 2002.

Vidal, M., Melgar, G.,'Spatial and temporal hydrochemical changes in groundwater under the contaminating effects of fertilizers and wastewater", journal of environmental management, Vol.,60, N.3, Nov., 2000.

\title{
ENVIRONMENTAL AWARENESS OF FARMERS IN GERNADA AND ANDOLA IN AL.GABAL AL AKHTAR LIBYA
}

\author{
Abdel Shafy A.A. Azam
}

\section{ABSTRACT:}

This study aimed to determining the farmers environmental awareness. to determine relationship between the degree of Farmers Environmental Awareness and some studied independent variables to contribution of each variable of these independent variables

Fayoum J. Agric. Res. \& Dev., Vol.24, No.2, July, 2010 
The study conducted in Gernada and Gandola Al.Gabal Al-Akhdar region on 120 of Farmers as systematic random sample equal to 50\%. Using questionnaire form prepared for this purpose. Data was collected by personal interview.

Statistical methods for analyzing data and testing hypotheses was simple correlation co efficient, multiple correlation and regression analysis, means, percentages.

The results revealed that:

The farmers Environmental Awareness level was low.

simple correlation co efficient revealed that: five independent variables was significant of Environmental Awareness level. multiple correlation and regression analysis revealed that: four independent variables was significantly associated with degree of Environmental Awareness level, that these variables jointly contributed to $45 \%$.these variables were: degree of education. degree of social participation, degree of cosmopolite, degree of information sources.

The results revealed that neighbors, and friends was the most popular Environmental information sources, T.V. was the second, personal experience was the third, faculty of agriculture came at the end as the least proportion of the respondents mentioned it as an Environmental information sources.

The Environmental training program aspects were determined on the basis of the actual needs and the respondents characteristics.

Fayoum J. Agric. Res. \& Dev., Vol.24, No.2, July, 2010 